\title{
Double Charge Exchange on Medium Mass Nuclei
}

\author{
Huachuan $\mathrm{Wu}$ \\ Instituto de Fisica, Universidad de Antioquia, Medellin, Colombia \\ and William R. Gibbs \\ Physics Department, New Mexico State University, Las Cruces, NM 88003, USA
}

Received on 22 September, 2003

\begin{abstract}
We studied the double-charge-exchange reaction on ${ }^{93} \mathrm{Nb}$ and ${ }^{56} \mathrm{Fe}$ targets. The energy dependence of forward transition was calculated based on an optical model with the nuclear structure described in the pseudo-SU(4) model.
\end{abstract}

\section{Introduction}

The systematic data on the forward DCX reaction show a peak at low energy (around $50 \mathrm{MeV}$ ), for both the doubleisobaric-analog state (DIAS) and the ground state (GS) transitions [1]. There have been controversial theoretical explanations of this peak. While some authors [2] have claimed that the peak is evidence for the existence of a dibaryon, we explained [3, 4] this peak as the consequence of pion distortion based on a two-step sequential process which involves nucleons solely. It has been also shown that the energy dependence of the forward transition of DCX is sensitive to the configuration mixing [4].

The purpose of present work is to extend our previous studies $[3,4]$ to medium mass nuclei, for which calculations are rare. To be specific, we study the DCX reactions ${ }^{56} \mathrm{Fe}\left(\pi^{+}, \pi^{-}\right){ }^{56} \mathrm{Ni}$ and ${ }^{93} \mathrm{Nb}\left(\pi^{+}, \pi^{-}\right){ }^{93} \mathrm{Tc}$. Data show that the forward transition also has a peak around $50 \mathrm{MeV}$ $[1,5,6]$.

For the nuclear structure of these nuclei a huge shell model space is needed, which makes an exact shell model calculation almost impossible. However, as one often does for heavy nuclei, the use of appropriate symmetry models can reduce the space substantially, while keeping the most important physics. The pseudo-SU(4) model [7] was proposed to deal with the nuclei in the $2 \mathrm{p}_{\frac{1}{2}}-2 \mathrm{p}_{\frac{3}{2}}-1 \mathrm{f}_{\frac{5}{2}}$ shell (hereafter denoted as the pf-shell). Although the nuclei ${ }^{93} \mathrm{Nb}$ and ${ }^{56} \mathrm{Fe}$ are not pf-shell nuclei, in this work, we use this model to describe parts of the nuclear configurations.

\section{Nuclear Structure}

In an early work [8] the structure of ${ }^{93} \mathrm{Nb}$ was described by $\mathrm{a}^{88} \mathrm{Sr}$ core plus three protons in the $2 \mathrm{p}_{\frac{1}{2}}-1 \mathrm{~g}_{\frac{9}{2}}$ orbits and two neutrons in the $2 \mathrm{~d}_{\frac{5}{2}}-3 \mathrm{~s}_{\frac{1}{2}}$ orbits, respectively. In this work we intend to extend the configuration space by taking advantage of the pseudo-SU(4) model.

The pseudo-SU(4) model restores symmetry in the nuclei with large 1-s coupling by adopting the concept of pseudo-spin and pseudo-orbits [9]. Since the pseudo-spinpseudo-orbit splitting (between $2 \tilde{\mathrm{d}}_{\frac{3}{2}}$ and $2 \tilde{\mathrm{d}}_{\frac{5}{2}}$, where tilde means the pseudo-orbit) is substantially smaller than the normal spin-orbit splitting (between $2 \mathrm{p}_{\frac{3}{2}}$ and $2 \mathrm{p}_{\frac{1}{2}}$ ), the violation of the pseudo-SU(4) is corresponding smaller. The advantage of the pseudo-SU(4) symmetry is that it keeps all the algebraic features of the SU(4) model while incorporating large 1-s coupling. A brief analysis in ref. [7] indicates that the pseudo-SU(4) model is promising for describing the pf-shell nuclei. Here we apply this model for the wave functions of the nucleons which are in the pf-shell.

First we discuss the structure of ${ }^{93} \mathrm{Nb}$. Without changing the actual nuclear configuration we use ${ }^{90} \mathrm{Zr}$ as the core. The protons and neutrons outside the core are in different major shells, and we can write the wave function of the nucleus ${ }^{93} \mathrm{Nb}$ as follows:

$$
\left|\psi\left({ }^{93} \mathrm{Nb}\right)>=\left\{\sqrt{1-\alpha_{1}^{2}}\left|\left(1 \mathrm{~g}_{\frac{9}{2}}\right)^{1}>_{p}+\alpha_{1}\right|(\mathrm{pf})^{-2}\left(\lg _{\frac{9}{2}}\right)^{3}>_{p}\right\}\right|(\mathrm{ds})^{2}>_{n},
$$

where $\alpha_{1}$ should be determined by an independent study of nuclear structure, however, in this work, as a trial, we take it as a free parameter. The subscripts $p$ and $n$ denote the proton and neutron configurations, respectively. We further assume the pseudo-SU(3) symmetry for the pseudo-orbitals, and therefore the configurations $(\mathrm{pf})^{-2}$ and $(\mathrm{ds})^{2}$ can be derived as follows:

$$
\left|(\mathrm{pf})^{-2}>=\frac{\sqrt{5}}{3}\right|\left(2 \mathrm{p}_{\frac{1}{2}}\right)^{-2}>+\frac{2}{3} \sqrt{\frac{2}{5}}\left|\left(2 \mathrm{p}_{\frac{3}{2}}\right)^{-2}>+\frac{2}{3} \sqrt{\frac{3}{5}}\right|\left(1 \mathrm{f}_{\frac{5}{2}}\right)^{-2}>
$$


and

$$
\left|(\mathrm{ds})^{2}>=\frac{\sqrt{5}}{3}\right|\left(3 \mathrm{~s}_{\frac{1}{2}}\right)^{2}>+\frac{2}{3} \sqrt{\frac{2}{5}}\left|\left(2 \mathrm{~d}_{\frac{3}{2}}\right)^{2}>+\frac{2}{3} \sqrt{\frac{3}{5}}\right|\left(2 \mathrm{~d}_{\frac{5}{2}}\right)^{2}>.
$$

Note that in the wave function of eq. 1 the ${ }^{90} \mathrm{Zr}$ core is neglected. This wave function extends the configuration space of ref. [8] to including also the $2 \mathrm{~d}_{\frac{3}{2}}$ for neutron particles and the $2 \mathrm{p}_{\frac{3}{2}}-1 \mathrm{f}_{\frac{5}{2}}$ for proton holes. As was shown in our previous work [4] this extension of configuration space is significant since the DCX forward transition is sensitive to the configuration mixing.

The wave function for ${ }^{93} \mathrm{Tc}$ is assumed to have a structure similar to ${ }^{93} \mathrm{Nb}$, and it can be expressed as,

$$
\left|\psi\left({ }^{93} \mathrm{Tc}\right)>=\sqrt{1-\alpha_{2}^{2}}\right|\left(\lg _{\frac{9}{2}}\right)^{3}>_{p}+\alpha_{2} \mid(\mathrm{pf})^{-2}\left(\lg _{\frac{9}{2}}\right)^{5}>_{p},
$$

where the structure of the (pf $)^{-2}$ configuration is the same as eq. 2 . While ${ }^{56} \mathrm{Ni}$ is thought to be a doubly closed shell nucleus, the structure of ${ }^{56} \mathrm{Fe}$ can be described as,

$$
\left|\psi\left({ }^{56} \mathrm{Fe}\right)>=\left\{\sqrt{1-\alpha_{3}^{2}}\left|\left(1 \mathrm{f}_{\frac{7}{2}}\right)^{-2}>_{p}+\alpha_{3}\right|(\mathrm{ds})^{-2}>_{p}\right\}\right|(\mathrm{pf})^{2}>_{n},
$$

where an excitation from the closed ds shell is included and $\alpha_{3}$ is taken as a parameter. Note, in eq. 5 the ${ }^{56} \mathrm{Ni}$ core is neglected.

To demonstrate the effects of configuration mixing on the DCX reaction, we also make a comparison between the calculation with the symmetry model wave functions (as expressed in eqs 1, 4 and 5) and that by using the single particle shell model (SPSM) wave function. The SPSM wave functions of ${ }^{93} \mathrm{Nb},{ }^{93} \mathrm{Tc}$ and ${ }^{56} \mathrm{Fe}$ are

$$
\left|\left(\mathrm{g}_{\frac{9}{2}}\right)^{1}>_{p}\right|\left(2 \mathrm{~d}_{\frac{5}{2}}\right)^{2}>_{n}, \mid\left(1 \mathrm{~g}_{\frac{9}{2}}\right)^{3}>_{p}, \text { and }\left|\left(1 \mathrm{f}_{\frac{7}{2}}\right)^{-2}>_{p}\right|\left(2 \mathrm{p}_{\frac{3}{2}}\right)^{2}>_{n}
$$

respectively.

\section{Energy Dependence of Forward DCX}

The calculation of the DCX reaction follows the procedure developed in ref. [10]. In our previous work [3, 4] the parameters of the optical model are taken from the fits to elastic scattering. However, for the targets studied in this paper, there exist no such fits. As a trial we use the optical model parameters of ${ }^{90} \mathrm{Zr}$ for ${ }^{93} \mathrm{Nb}$, and by interpolating the opti- cal parameters of ${ }^{40} \mathrm{Ca}$ and ${ }^{90} \mathrm{Zr}$ we get the optical model parameters of ${ }^{56} \mathrm{Fe}$. The optical model parameters are listed in Table 1. Note that the ranges, i.e. S11,..., P33, are in $\mathrm{MeV}$.

Another important ingredient in the calculation is the single particle wave function, especially the wave function of those orbits which are involved in the DCX reaction. The single particle wave functions are calculated by using a Woods-Saxon type of potential, the parameters being so adjusted that both the separation energy of the proton and neutron and the rms proton radius match the experimental values.

TABLE I: Optical model parameters

\begin{tabular}{|c|c|c|c|c|c|c|c|c|c|}
\hline Nucleus & S11 & S31 & P11 & P31 & P13 & P33 & $\begin{array}{c}\text { s wave } \\
\text { multiplier }\end{array}$ & $\begin{array}{c}\text { p-wave } \\
\text { multiplier }\end{array}$ & absorption \\
\hline${ }^{93} \mathrm{Nb}$ & 404 & 497 & 277 & 210 & 253 & 1173 & 1.21 & 1.14 & 1.00 \\
\hline${ }^{56} \mathrm{Fe}$ & 216 & 506 & 303 & 215 & 187 & 1128 & 1.20 & 1.09 & 0.59 \\
\hline
\end{tabular}


Figures 1 and 2 present the preliminary results of the energy dependence of DCX on ${ }^{93} \mathrm{Nb}$ and ${ }^{56} \mathrm{Fe}$, respectively. In both figures, the solid line represents the result of the symmetry model wave function, and the dashed line gives the result of the SPSM wave function. A fair agreement is achieved for both the DCX reactions, at least in the low energy region. The peak at around $50 \mathrm{MeV}$ appears as the consequence of the pion distortion without invoking explicit degrees of freedom of a dibaryon. However, in the resonance region there exist discrepancies. For example, while the experimental data show a peak around 150-160 $\mathrm{MeV}$, the theoretical prediction increases with energy almost monotonously. This might suggest that inclusion of explicit degrees of freedom of non-nucleons is necessary.

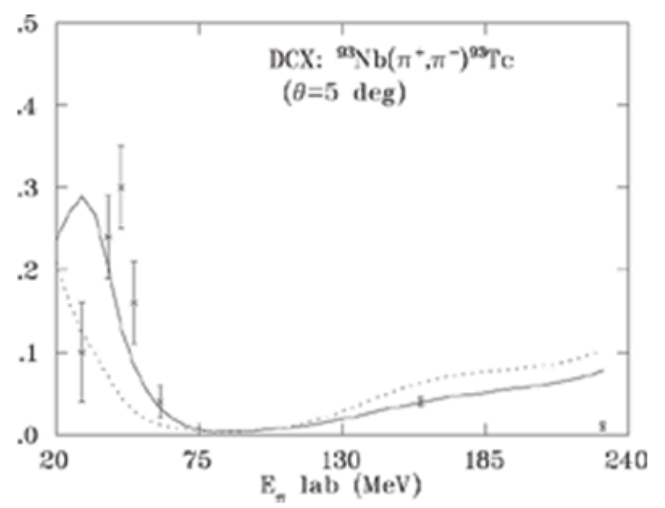

Figure 1. Energy dependence of forward transition of DCX on ${ }^{93} \mathrm{Nb}$. The solid line represents the result of the nuclear wave function given in eq. 1 and 4 , and the dashed line gives the result of single particle shell model.

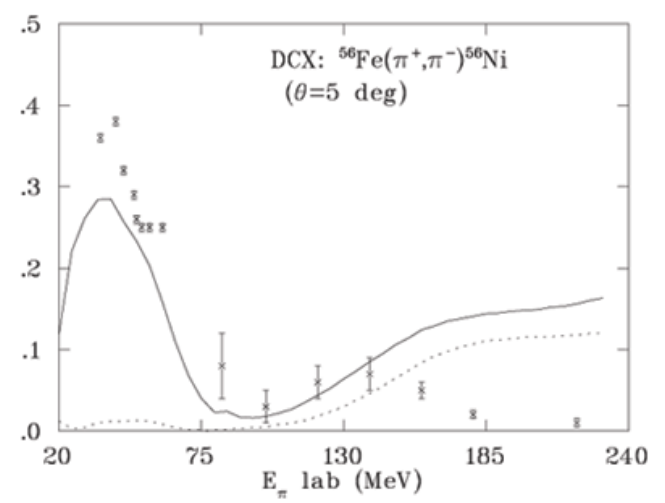

Figure 2. Energy dependence of forward transition of DCX on ${ }^{93} \mathrm{Nb}$. The solid line represents the result of the nuclear wave function given in eq. 5 , the dashed line gives the result of single particle shell model.

A comparison between the results of the symmetry model wave function (solid lines) and those of SPSM (dashed line) shows that a proper configuration mixing is very important to reproduce data. We note that the mixing ratios $\alpha_{1}, \alpha_{2}$ and $\alpha_{3}$ are tentatively taken as 0.35 , however, an independent nuclear structure study is needed to determine these ratios.

\section{Conclusion and Discussion}

This work presents preliminary results of the energy dependence of forward DCX reactions (the GS transition) on ${ }^{93} \mathrm{Nb}$ and ${ }^{56} \mathrm{Fe}$ targets. The calculation reproduces experiments fairly well, at least in the low energy region. In this calculation the theoretical framework is a conventional sequential process, and the peak at low energy arises naturally as the consequence of pion wave distortion.

As was indicated in our previous study that the DCX reaction is sensitive to the configuration mixing in the nuclear structure. In this work we use two types of wave functions, i.e. the pseudo-SU(4) model wave function and that of the single particle shell model. A comparison between the DCX calculations with these two types of wave functions shows that the configuration mixing in the nuclear wave function is important in reproducing data. In other words, the energy dependence of the forward DCX reaction contains information not only on the fundamental charge exchange process but on the nuclear structure as well.

We note that the value (0.35) of the mixing ratios $\alpha_{1}, \alpha_{2}$ and $\alpha_{3}$ is only tentative. An independent nuclear structure study is underway in order to derive these mixing ratios. The possible change of these mixing ratios will certainly cause some change in the energy dependence of the forward DCX amplitude, however, the qualitative features (for example, the peak around $50 \mathrm{MeV}$ ) as shown in this article will remain.

\section{Acknowledgment}

The authors thank the University of Antioquia, Colombia, and the National Science Foundation (under contract PHY-0099729) for financial support.

\section{References}

[1] J. Draeger et al., Phys. Rev. C 62, 064615 (2000).

[2] R. Bilger, H. Clement, and M.G. Schepkin, Phys. Rev. Lett. 71, 42 (1993); 72, 2972 (1994).

[3] M. Nuseirat, M.K. Lodhi, M.O. El-Ghossain, W.R. Gibbs, and W.B. Kaufmann, Phys. Rev. C58, 2292 (1998).

[4] H.C. Wu and W.R. Gibbs, Phys. Rev. C 68, 054610 (2003).

[5] J. Pätzold et al., Phys. Lett. B428, 18 (1998).

[6] R. Bilger et al., Phys. Lett. B269, 247 (1991).

[7] P. Van Isacker, O. Juillet, and F. Nowacki, Phys. Rev. Lett. 82, 2060 (1999).

[8] A.M. Van den Berg, E.A. Bakkum, P.W.M. Glaudemans, and D. Zwarts, Phys. Rev. C 29, 1109 (1984).

[9] A. Arima, M. Harvey, and K. Shimizu, Phys. Lett. B 30, 67 (1969).

[10] H.C. Wu and W.R. Gibbs, Phys. Rev. C62 044614 (2000). 